Mens

Revue d'histoire intellectuelle et culturelle

\title{
Réplique au compte rendu de Dominique Marquis
}

\section{Martin Roy}

Volume 16, numéro 1, automne 2015

URI : https://id.erudit.org/iderudit/1038987ar

DOI : https://doi.org/10.7202/1038987ar

Aller au sommaire du numéro

Éditeur(s)

Centre de recherche en civilisation canadienne-française

ISSN

1492-8647 (imprimé)

1927-9299 (numérique)

Découvrir la revue

Citer ce document

Roy, M. (2015). Réplique au compte rendu de Dominique Marquis. Mens, 16(1), 143-147. https://doi.org/10.7202/1038987ar d'utilisation que vous pouvez consulter en ligne.

https://apropos.erudit.org/fr/usagers/politique-dutilisation/ 


\title{
Réplique
}

\section{Réplique au compte rendu de Dominique Marquis}

\author{
Martin Roy \\ Auteur d'Une réforme dans la fidélité
}

La recension qu'a faite Dominique Marquis d'Une réforme dans la fidélité est assez négative ${ }^{1}$. Répondons méthodiquement, donc, aux remarques étonnantes de l'auteure.

Les premières critiques concernent l'affaire Bradet. D'un côté, l'auteure reproche à mon exposé sur les causes de cette affaire une «certaine ambiguïté » (p. 262). La décision des autorités dominicaines de renvoyer le père Bradet contredirait mon affirmation relative à la très grande marge de manœuvre éditoriale dont aurait bénéficié la revue. Présenter les choses ainsi est réducteur. D’abord, ce congédiement n'était dû qu'à des circonstances exceptionnelles. Les autorités dominicaines, qui, en temps normal, accordaient une grande liberté à Maintenant, n'ont renvoyé le père Bradet que sous la pression du général des dominicains qui, conservateur, était mécontent de la revue. Ce renvoi exigé de Rome intervenait dans un contexte particulier alors que la minorité conservatrice à Vatican II voulait contrer l'influence de la majorité conciliaire dans la catholicité. Soumises à de telles pressions, les autorités canadiennes de l'Ordre ont cru nécessaire d'obtempérer. La mise au pas de la revue empêchée par

${ }^{1}$ Mens : revue d'histoire intellectuelle et culturelle, vol. XIV, $\mathrm{n}^{\circ} 2$-vol. XV, $\mathrm{n}^{\circ} 1$ (printemps-automne 2014), p. 261-264. 
l'opinion publique, le mensuel allait jouir de nouveau, après cet épisode, d'une grande autonomie éditoriale. D'un autre côté, l'auteure ne croit pas qu'à ce sujet il soit " pertinent d'utiliser le concept d'opinion publique» (p. 263). L'auteure insinue que cette controverse n'intéressait qu'une poignée d'intellectuels catholiques. Mais comment interpréter, par exemple, l'abondance du courrier des lecteurs des grands quotidiens, relativement à cette histoire, de l'aveu même des rédactions? L'Église ayant connu, par le passé, plusieurs " affaires ", l'opinion publique craignait, au moment où la catholicité faisait peau neuve, tout retour en arrière de la part d'une institution encore engoncée dans son autoritarisme et son cléricalisme. Le cas Bradet faisait donc figure de symbole. Comme événement sans portée, donc, on a déjà vu mieux.

Ensuite, l'auteure m'impute le fait d'avoir trop parlé du contenu politique de Maintenant, et pas assez de ses pages culturelles. Pour elle, "la "mise à jour" du catholicisme ne pass[ait] pas uniquement par la/le politique» (p. 263). Cette critique m'inspire deux commentaires. D'abord, ai-je prétendu avoir écrit l'ouvrage définitif sur Maintenant? Non. Conscient que tout n'avait pas été dit, j'ai même analysé dernièrement "Christianisme et nouvelle culture ", un texte-phare de Vincent Harvey paru dans Maintenant en 1971. Cela dit, beaucoup reste encore à faire en la matière. Ensuite, où ai-je dit que cette mise à jour passait uniquement par la/le politique? Nulle part. L'esprit de mon propos tourne autour de ce mot : "principalement ", plutôt qu' " uniquement ». Selon la revue, la vision traditionnelle de l'espérance chrétienne, qui, largement contestée, ne promettait essentiellement que le Ciel, était la cause principale du désintérêt de la jeunesse politisée envers la foi chrétienne. C'était le plus grave problème auquel la foi faisait face à l'époque. Une prise en compte des espérances politiques et temporelles s'imposait de toute urgence, pour Maintenant, afin de recrédibiliser le christianisme dans un contexte d'effervescence sociopolitique. J'ai donc attribué à la question politique l'importance qu'elle méritait. 
Enfin, l'auteure me blâme d'avoir négligé l'influence du personnalisme préconciliaire sur les chrétiens de Maintenant. D'abord, tout le monde sait que Vatican II a consacré plusieurs théologiens réformateurs (Marie-Dominique Chenu, Yves Congar et Henri de Lubac, entre autres) qui n'inspiraient jusque-là que méfiance. Bien que je sois donc conscient que Vatican II n'est pas sorti du néant, je confesse toutefois que la bibliographie du livre recensé omet de citer certaines études sur l'époque préconciliaire, à commencer par l'article de Dominique Marquis sur la Revue dominicaine ${ }^{2}$. Mais, ces omissions exceptées, on ne peut me reprocher sérieusement de ne pas avoir assez insisté sur le personnalisme, dans la mesure où, notamment, le contexte dans lequel s'inscrivait Maintenant était très différent. Vatican II libérant de manière inédite les consciences et la parole chrétiennes, l'ambiance théologique dans les colonnes de Maintenant a donc dépassé rapidement l'univers de pensée personnaliste, lequel a cédé la place, très tôt, à des figures intellectuelles avancées, comme Harvey Cox, auteur de The Secular City (1965), et Jurgën Moltmann, à qui l'on doit une Théologie de l'espérance.

L'auteure me critique également pour avoir écrit ce passage qui oublierait l'apport personnaliste : « Le traitement qu'on [les chrétiens de Maintenant] réserva à ces diverses questions (laïcité, idées politiques, contraception, etc.) dut beaucoup aux réformes conciliaires ainsi que, plus généralement, au désir de changement qui agitait le monde catholique dans les années 1960 et 1970 (p. 301)³. Il y a énormément à dire sur cette affirmation que l'auteure, à tort, juge excessive. Commençons par la laïcité. Vatican II a reconnu, dans deux documents marquants, la "juste autonomie des réalités terrestres " (Gaudium et spes, 1965) et le principe de la liberté religieuse (Dignitatis humanae, 1965). Ces documents ont marqué la fin officielle de l'idéal

2 Dominique Marquis, "La Revue dominicaine, 1915-1961 : un regard catholique sur une société en mutation ", Revue d'histoire de l'Amérique française, vol. 62, $\mathrm{n}^{\circ}$ 3-4 (hiver-printemps 2009), p. 407-427, [En ligne], [DOI: 10.7202/038520ar].

3 Marquis, compte rendu, Mens, p. 264. 
catholique de l'État confessionnel. À cet égard, Vatican II marque un virage décisif. Je ne dis pas que la défense de la liberté religieuse et la dénonciation du constantinisme n'avaient jamais été entreprises auparavant, mais seuls ces textes ont permis que ces idées soient défendues aussi franchement et inconditionnellement. Avec l'appui de tels documents, les chrétiens de Maintenant pouvaient accueillir encore plus ouvertement les nouvelles théologies de la sécularisation et procéder à la liquidation de l'idéal de chrétienté. Quant aux idées politiques de Maintenant, il est clair que Vatican II a réhabilité la / le politique dans le monde catholique. Au sein de celui-ci a suivi un activisme sociopolitique nouveau. On excédait fréquemment le cadre d'un réformisme prudent qui avait été souvent la particularité des catholiques d'ici. Ce radicalisme nouveau était manifeste chez Maintenant qui flirtait parfois avec le mythe révolutionnaire. La revue a pu se convertir par ailleurs au socialisme grâce à Vatican II et à l'encyclique Populorum progressio (1967), qui ont mis en sourdine l'antisocialisme catholique. Quant à la contraception, c'est affaire bien connue que jusqu'au concile et aux travaux de la commission pontificale chargée de réexaminer la doctrine en matière de régulation des naissances (1964-1967), peu de catholiques ont osé défendre la contraception et critiquer sa prohibition romaine. Il aura fallu le contexte particulier des années 1960 et l'« esprit du concile » pour libérer la parole. Auparavant, seules les méthodes Ogino et sympto-thermique étaient vraiment envisagées.

Considérant l'écart existant entre les modalités du projet de réforme de l'Église des chrétiens de Maintenant et des réformateurs canadiens-français d'après-guerre, on peut douter, là aussi, de l'utilité de disserter longuement sur une continuité supposément étroite entre ces deux projets. Alors que ces derniers n'avaient pas excédé, pour la plupart, une libéralisation interne de la chrétienté canadiennefrançaise, par le truchement d'une décléricalisation et d'une rationalisation accrues des institutions confessionnelles, les chrétiens de Maintenant ont milité pour une sortie complète de la chrétienté et une intégration des croyants aux nouvelles structures laïques. Ils se 
libéraient conséquemment d'un paradigme qui avait mis d'abord l'accent sur la différence et la spécificité catholiques, sur les plans institutionnel et cognitif. Compte tenu de cet écart et des autres raisons invoquées, je me suis donc limité à évoquer, sans plus, la notion d'une dette de Maintenant envers le personnalisme d'après-guerre. 